Association of Angiopoietin-2 and Ki-67 Expression with Vascular Density and Sunitinib Response in Metastatic Renal Cell Carcinoma

Rautiola, Juhana

2016-04-21

Rautiola , J , Lampinen , A, Mirtti , T , Ristimaki , A , Joensuu , H , Bono , P \& Saharinen , P 2016 , ' Association of Angiopoietin-2 and Ki-67 Expression with Vascular Density and Sunitinib Response in Metastatic Renal Cell Carcinoma ' , PLoS One , vol. 11 , no. 4 , 0153745 . https://doi.org/10.1371/journal.pone.0153745

http://hdl.handle.net/10138/163114

https://doi.org/10.1371/journal.pone.0153745

cc_by

publishedVersion

Downloaded from Helda, University of Helsinki institutional repository.

This is an electronic reprint of the original article.

This reprint may differ from the original in pagination and typographic detail.

Please cite the original version. 


\title{
Association of Angiopoietin-2 and Ki-67 Expression with Vascular Density and Sunitinib Response in Metastatic Renal Cell Carcinoma
}

\author{
Juhana Rautiola $^{1 \oplus}$, Anita Lampinen $^{2 \odot}$, Tuomas Mirtti $^{4,5}$, Ari Ristimäki ${ }^{5}$, Heikki Joensuu ${ }^{1}$, \\ Petri Bono ${ }^{1}$, Pipsa Saharinen ${ }^{2,3 *}$ \\ 1 Comprehensive Cancer Center, Helsinki University Hospital, P.O.B. 180, 00029 HUS, Finland and \\ University of Helsinki, Finland, 2 Translational Cancer Biology Program, Research Programs Unit, and \\ Department of Virology, Haartman Institute, Biomedicum Helsinki, Haartmaninkatu 8, P.O.B. 63, FI-00014, \\ University of Helsinki, Finland, 3 Wihuri Research Institute, Biomedicum Helsinki, Haartmaninkatu 8, FI- \\ 00290, Helsinki, Finland, 4 Institute for Molecular Medicine Finland, Haartmaninkatu 8, P.O.B. 63, FI-00014, \\ University of Helsinki, Finland, 5 Pathology, Research Programs Unit and HUSLAB, University of Helsinki \\ and Helsinki University Hospital, P.O.B. 400, FI-00029, HUS, Helsinki, Finland
}

ه These authors contributed equally to this work.

*Pipsa.Saharinen@Helsinki.fi

\section{f open ACCEss}

Citation: Rautiola J, Lampinen A, Mirtti T, Ristimäki A, Joensuu $H$, Bono P, et al. (2016) Association of Angiopoietin-2 and Ki-67 Expression with Vascular Density and Sunitinib Response in Metastatic Renal Cell Carcinoma. PLoS ONE 11(4): e0153745. doi:10.1371/journal.pone.0153745

Editor: Samir M. Parikh, Beth Israel Deaconess Medical Center, UNITED STATES

Received: November 29, 2015

Accepted: April 4, 2016

Published: April 21, 2016

Copyright: $\odot 2016$ Rautiola et al. This is an open access article distributed under the terms of the Creative Commons Attribution License, which permits unrestricted use, distribution, and reproduction in any medium, provided the original author and source are credited.

Data Availability Statement: All relevant data are within the paper and its Supporting Information files.

Funding: Funded by https://www.cancersociety.fi/ grants/cancer-foundation-grants/; Cancer Foundation (PS) and 271845; https://www.aka.fi; Academy of Finland Centre of Excellence Program 2014-2019 (to PS, HJ). The funders had no role in study design, data collection and analysis, decision to publish, or preparation of the manuscript.

\section{Abstract}

The Angiopoietin-2 (Ang2, Angpt2) growth factor is a context-dependent antagonist/agonist ligand of the endothelial Tie2 receptor tyrosine kinase and known to promote tumour angiogenesis and metastasis. Angiopoietin antagonists have been tested in clinical cancer trials in combination with VEGF-based anti-angiogenic therapy, including sunitinib, which is widely used as a first-line therapy for metastatic renal cell carcinoma (mRCC). However, little is known about Ang2 protein expression in human tumours and the correlation of tumour Ang2 expression with tumour vascularization, tumour cell proliferation and response to antiangiogenic therapies. Here, we evaluated, using immunohistochemistry, the expression of Ang2, CD31 and the cell proliferation marker Ki-67 in the primary kidney cancer from 136 mRCC patients, who received first-line sunitinib after nephrectomy. Ang2 protein expression was restrained to RCC tumour vessels, and correlated with tumour vascularization and response to sunitinib. High pre-therapeutic Ang2 expression, and more strongly, combined high expression of both Ang2 and CD31, were associated with a high clinical benefit rate (CBR). Low cancer Ki-67 expression, but not Ang2 or CD31 expression, was associated with favourable progression-free (PFS) and overall survival (OS) as compared to patients with high Ki-67 expression (PFS 6.5 vs. 10.6 months, $P=0.009$; OS, 15.7 vs. 28.5 months, $P=0.015)$. In summary, in this study to investigate endothelial Ang2 in mRCC patients treated with first-line sunitinib, high cancer Ang2 expression was associated with the CBR, but not PFS or OS, whereas low Ki-67 expression was significantly associated with long PFS and OS. 
Competing Interests: PB has received honoratia from Novartis, Pfizer, Bms, Msd, and Orion Pharma, and research funding from Novartis.

\section{Introduction}

Renal cell carcinoma (RCC) represents approximately two to three per cent of adult malignancies worldwide, and has an increasing rate of incidence in many countries [1]. The majority of RCCs are classified as clear cell (80\%) and papillary cancers (10\%), which are thought to arise in the epithelium of the proximal tubules [2]. In approximately $60-75 \%$ of sporadic clear cell RCCs (ccRCC), the von Hippel-Lindau (VHL) tumour suppressor gene is inactivated, resulting in stabilization of the hypoxia inducible factors (HIFs), which regulate metabolic and vascular tumour responses, including increased expression of the HIF target vascular endothelial growth factor (VEGF) [3-5].

The prognosis of metastatic RCC (mRCC) is poor, and the disease is notoriously resistant to chemotherapy, with a minority of the patients responding to traditional immunotherapy, such as interferon and interleukin-2 [1,6]. Since 2005, seven novel targeted therapies inhibiting the VEGF signalling pathway and the mammalian target of rapamycin (mTOR) have been approved for the treatment of mRCC, but complete durable responses with any of the targeted therapies are rare [7].

Sunitinib, a multi-targeted tyrosine kinase inhibitor of VEGF receptors (VEGFRs), PDGFRa/b, KIT, Flt-3 and CSF-1R, is widely used as a first-line therapy for mRCC [8]. Objective response rates of $25-47 \%$ have been reported to sunitinib in mRCC [9]. However, disease progression usually occurs about 11 months after the initiation of sunitinib treatment [9]. Furthermore, $10-20 \%$ of patients exhibit no clinical benefit [9], [10]. Preclinical studies have identified potential mechanisms behind the development of acquired resistance to anti-angiogenic therapy, including adaptive changes in the activation of VEGF-independent angiogenic pathways, altered cellular metabolism and the activation of cancer stem cells [11-13]. However, the mechanisms of intrinsic resistance, where patients do not benefit at all from VEGF-targeted drugs remain largely unknown [8]. Thus, there is a need for biomarkers predicting sunitinib response and for identifying patients who will benefit from the therapy, but so far no such established markers are in clinical use [14].

Angiopoietin-2 (Ang2, Angpt2) is an endothelial cell-derived growth factor, which binds in an autocrine fashion to the endothelial Tie2 receptor tyrosine kinase on blood and lymphatic vessels [15]. Ang2 is expressed at low levels during normal homeostasis, but at increased levels in many human diseases characterized by increased vascular leak and inflammation, such as sepsis [16], and in cancer, including RCC [17-19]. Pre-clinical studies have demonstrated tumour growth and angiogenesis inhibition using Ang2 blocking reagents, especially, when combined with VEGF-based anti-angiogenic therapies [12,20-23] as well as inhibition of lymph node and distant metastasis $[21,24,25]$. Angiopoietin antagonists, which are in the clinical development have been combined with paclitaxel in phase III ovarian cancer trials [26], and more recently, with sunitinib in a phase II mRCC trial [27], but more studies are needed to evaluate the benefit of angiopoietin blocking in human cancer.

Ang2 mRNA expression has been reported to predict poor prognosis in breast cancer [28], and high circulating Ang2 levels were reported to predict unfavourable outcome in metastatic colorectal carcinoma [29], mRCC [30] and melanoma [31]. However, few studies have so far directly investigated Ang2 protein expression in human tumour tissues, mainly due to lack of reliable immunohistochemical methods. Thus, the cell type expressing Ang2 in RCC tumour tissues has not been identified and the potential significance of Ang2 protein expression in RCC for tumour angiogenesis, tumour cell proliferation or response to anti-angiogenic therapies remains unknown.

$\mathrm{Ki}-67$ is a large nuclear protein, which has prognostic relevance in many malignant diseases, including local RCC $[32,33]$. High expression of Ki-67 in patients with local RCC nearly 
doubled the risk of death [33]. However, Ki-67 has not been largely investigated as a prognostic or predictive factor in $\mathrm{mRCC}$ patients treated with angiogenesis inhibitors.

The purpose of this study was to investigate pre-therapeutic Ang2, Ki-67 and CD31 expression in the primary human RCC tissue in patients with metastatic disease, the correlation of baseline Ang2 and Ki-67 expression with tumour vascular content and the relation of their expression to the outcome of first-line sunitinib treatment in patients with mRCC. Using 136 pre-therapeutic samples from primary mRCC tumours, we found that Ang2 is selectively expressed in the tumour vasculature, but not in RCC tumour cells. Baseline Ang2 expression correlated with vascular density, but not with PFS or OS, although high Ang2 expression was associated with clinical benefit to sunitinib. Low expression of Ki-67 correlated significantly with prolonged PFS and OS, suggesting that tumour cell proliferation is a potential prognostic marker for first-line sunitinib therapy in mRCC.

\section{Materials and Methods}

\section{Patients, Treatment and Assessment of Tumour Response}

A total of 181 consecutive patients with mRCC were treated with sunitinib at the Department of Oncology of Helsinki University Central Hospital (HUCH) between October 18, 2006 and May 31, 2012. Of these patients histological specimens could be retrieved from 136 patients, and data about response to sunitinib treatment was available from 126 patients. The main reason for the lack of efficacy data in the remaining ten cases was too short treatment to be evaluable. Patients were identified from the hospital registry and the clinical data were collected from the hospital case records. We required for the study inclusion that the patients had histologically identified mRCC treated with sunitinib as their first systemic treatment for advanced disease. No prior targeted cancer therapies were allowed.

Following the approval of the study protocol by a HUCH Ethics Committee, the formalinfixed paraffin-embedded samples containing tumour tissue were collected from the archives of the Department of Pathology, HUCH. HUCH ethics committee waived the need for individual consent, since all data were analyzed anonymously, and approved this retrospective study. The cut-off date for the data capture was June 1,2012. The features of the study patient population are provided in Table 1. The median duration of sunitinib treatment was 7.2 months (range 0.23 to 65.3 months).

None of the patients had received prior tyrosine kinase inhibitor therapy, but 18 (13\%) had received prior interferon- $\alpha$. Sunitinib was administered until disease progression, death or until toxicity was considered unacceptable. Of the 136 patients, 43 patients had $25 \mathrm{mg} /$ day continuous daily dosing (CDD) as their starting dose, 61 patients had $37.5 \mathrm{mg} /$ day CDD and 32 patients had $50 \mathrm{mg} /$ day 4 weeks on and 2 weeks off, the latter two dosages being widely accepted and used as global standards for the treatment of mRCC [34].

Response to treatment was assessed using computed tomography (CT) performed at 8 to 12 week intervals after starting sunitinib. A professional radiologist interpreted all images. Treatment efficacy was reported according to Response Evaluation Criteria in Solid Tumours v. 1.0 (RECIST).

\section{Histology and Immunohistochemistry}

Five $\mu \mathrm{m}$ thick sections were cut from formalin-fixed, paraffin-embedded tumour tissues and stained using haematoxylin and eosin for histological analysis. Immunohistochemistry (IHC) was performed on deparaffinised (with xylene) and rehydrated sections by using the Tyramide Signal Amplification kit (PerkinElmer, Waltham, MA, USA) following the manufacturer's instructions. Antigen retrieval was conducted in $10 \mathrm{mM}$ citrate buffer using microwaves at 750 
Table 1. Patient Characteristics.

\begin{tabular}{ll}
\hline & No. (\%) \\
\cline { 2 - 2 } & (N = 136) \\
\hline Sex & $79(58)$ \\
Male & $57(42)$ \\
Female & \\
WHO performance status & $77(57)$ \\
0 & $59(43)$ \\
\hline 1 & $135(99)$ \\
Prior nephrectomy & \\
Reason for stopping sunitinib & $81(60)$ \\
Progression & $55(40)$ \\
Adverse events/other & \\
Heng risk group & $67(49)$ \\
Favourable & $30(22)$ \\
Intermediate & $35(26)$ \\
Poor & $4(3)$ \\
N.A. & \\
MSKCC risk group & $22(16)$ \\
Favourable & $81(60)$ \\
Intermediate & $22(16)$ \\
Poor & $11(8)$ \\
N.A. & $120(88)$ \\
Histological type & $12(9)$ \\
\hline Clear cell & $4(3)$ \\
\hline Papillary & \\
\hline Other & \\
\hline
\end{tabular}

Abbreviations: WHO, World Health Organization; N.A, not available; MSKCC, Memorial Sloan-Kettering Cancer Center

doi:10.1371/journal.pone.0153745.t001

$\mathrm{W}$ for $5 \mathrm{~min}$, and subsequently at $450 \mathrm{~W}$ for $10 \mathrm{~min}$. Endogenous peroxidase activity and nonspecific binding sites were blocked using $10 \%$ hydrogen peroxidase and a TNB (0.5\%) blocking buffer (PerkinElmer), respectively. The sections were incubated with goat polyclonal antibodies to amino acid residues Asp68-Phe496 of human Angiopoietin-2 (1:50, $0.2 \mathrm{mg} / \mathrm{ml}$, R\&D Systems, Minneapolis, MN) and with a mouse monoclonal antibody to human CD31 (1:100, 0.2 $\mathrm{mg} / \mathrm{ml}$, JC70A, DAKO, Glostrup, Denmark), diluted in TNB overnight at $+4^{\circ} \mathrm{C}$, washed with TNT buffer (100 mM Tris pH 7.4, $150 \mathrm{mM} \mathrm{NaCl}, 0.05 \%$ Tween-20), and subsequently incubated with biotinylated anti-goat or anti-mouse secondary antibodies $(1: 300,1.5 \mathrm{mg} / \mathrm{ml}$, Vector Laboratories Inc, Burlingame, CA) in TNB for $30 \mathrm{~min}$ at room temperature, washed and detected using a chromogenic visualizing method AEC (3-amino-9-ethylcarbazole) for $8 \mathrm{~min}$. The sections were counterstained with Mayer's Hemalum Solution (Merck KGaA, Darmstadt, Germany) and mounted using Aquatex (Merck KGaA). To confirm the specificity of the Ang2 staining, Ang2 antibodies were incubated with 5x molar excess of recombinant human Ang2 $(3 \mu \mathrm{g})$ (R\&D Systems) in TNB for $20 \mathrm{~min}$, and subsequently used for staining of the tissue sections as above.

Immunostaining for Ki-67 was performed using a Ventana Bench Mark XT immunostainer automate (Ventana Medical Systems, Tucson, Arizona, USA) and a mouse anti-human Ki-67 
mAb (MIB-1, DAKO) with a Ventana UltraView Dab V3 (Ventana Medical Systems) kit with amplification.

\section{Scoring of Ang2, CD31 and Ki-67 Protein Expression}

The investigators scored independently the protein expression of Ang2 (JR+AL and PS), CD31 $(J R+A L)$ and $\mathrm{Ki}-67(J R+A L$ and TM). A general consensus was sought whenever the scores differed between the investigators. The mean Ang2+ and CD31+ vessel densities were estimated based on three vascular hot-spot areas using an Olympus Bx50 microscope (Olympus Optical Co., Ltd, Tokyo, Japan) and a 10x objective (magnification x100). The samples were scored for Ang2 and CD31 expression using four expression scores of negative (0), weak (1), moderate (2) and high expression (3). The score 0 was defined as no positive vessels, score 1 as $1-49$ vessels, score 2 as $50-99$ vessels, and score 3 as $\geq 100$ positively stained vessels per one $10 x$ microscope field. For statistical analysis using the Fisher's exact test tumours with CD31 expression scores 3 , which showed a highly vascular phenotype $(\geq 100 \mathrm{CD} 31+$ vessels/one microscope field), were considered to form the CD31 high category ( $40 \%$ of samples), and the samples with a score $0-2$ ( $<100$ vessels/one microscope field) the CD31 low category (60\%). Due to the lower level of Ang2 expression, tumours with Ang2 expression scores 2 and 3 ( $\geq 50$ Ang2+ vessels/one microscope field) and the tumours with Ang2 expression scores 0 and 1 ( $<50$ Ang2 + vessels/one microscope field) were combined to form the Ang2 high ( $38 \%$ of the samples) and Ang2 low categories (62\%), respectively. The expression of Ki-67 was considered high when more than $10 \%$ of the tumor cell nuclei, measured in three different hot spot areas for each sample, expressed Ki-67 [35]. A pathologist reviewed tumour histology in each case.

Images were captured using a Leica DM LB microscope (Meyer Instruments, Inc, Houston, TX) attached to an Olympus DP50 colour camera using 10x (NA (numerical aperture) 0.25), 20x (NA 0.4) and 40x (NA 0.65) objectives. Magnification was x100, x200 and x400, respectively.

\section{Statistical Analysis}

Progression-free survival (PFS) was calculated according to the Kaplan-Meier method from the date of initiation of sunitinib treatment to the date of documented cancer progression or death, whichever occurred first. Patients alive without progression were censored on the time of the last follow-up visit. Overall survival (OS) was counted from the date of sunitinib initiation to the date or death, censoring patients alive. Survival between groups was compared with the log-rank test. Frequency tables were analysed using the Fisher's exact test or the $\chi^{2}$-test. All $P$-values are two-tailed. The statistical analyses were performed with the IBM SPSS Statistics software for Mac, version 20.0 (IBM Corporation, Armonk, N.Y.).

\section{Results}

\section{Angiopoietin-2 Expression in the Tumour Vasculature Correlates with Vascular Density}

The immunohistochemical analysis of primary mRCC tumour tissue showed that Ang2 was specifically expressed in endothelial cells of the tumour blood vessels, but not in the tumour cells. A 5-fold molar excess of recombinant Ang2 was used to neutralize the polyclonal antibody to Ang2. This efficiently abolished the Ang2 signal in the tumour blood vessels confirming the specificity of the staining (Fig 1).

The analysis of $136 \mathrm{mRCC}$ samples showed consistent Ang2 expression in the tumour vessels, and variation in the levels of Ang2 expression between the samples (ranging from 0 to 

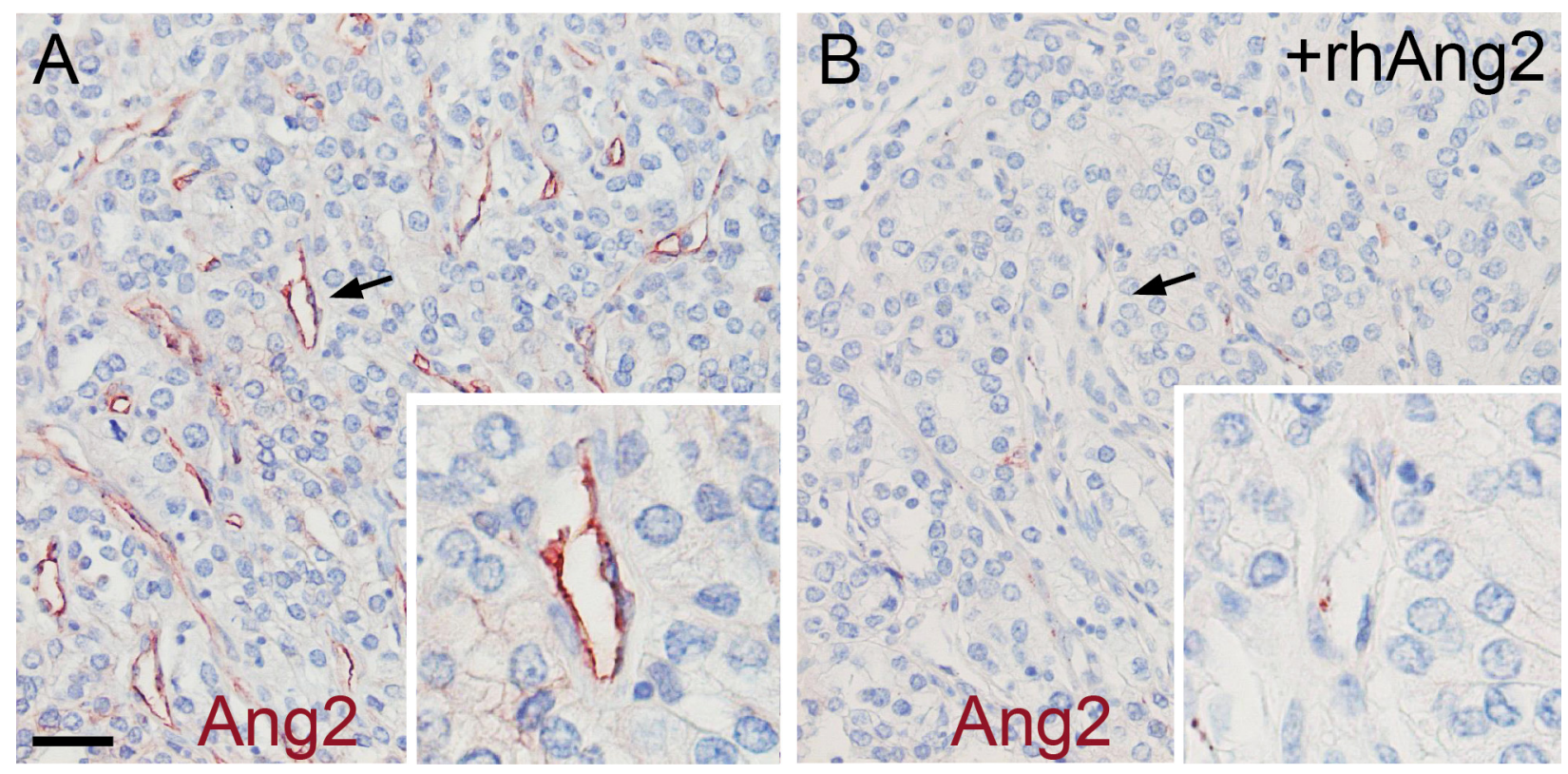

Fig 1. Representative immunohistochemical staining of Ang2 in mRCC. (A) immunohistochemical staining of paraffin-embedded mRCC primary tumour tissue using a polyclonal antibody to Ang2. The CD31 expression was scored 3 in the sample. (B) An adjacent section was stained using the polyclonal antibody to Ang2, blocked with a 5-fold molar excess of recombinant human Ang2 (+rhAng2). Arrows indicate the magnified areas. Magnifications x200 and $\mathrm{x} 400$. Scale bar $40 \mu \mathrm{m}$.

doi:10.1371/journal.pone.0153745.g001

$>100$ Ang2 positive vessels/microscopic field, median 5) (Fig 2A, S1A and S1C Fig). Occasionally, Ang2 expression was detected in the blood vessels of the glomeruli and in the tubular epithelium in the adjacent normal kidney tissue, and in the blood vessels of the associated fat tissue (not shown).

The tumours, analysed by immunohistochemical staining for CD31, showed variation in the vessel numbers (ranging from 0 to $>100 \mathrm{CD} 31$ positive vessels/microscope field, median 80) (Fig 2B, S1B and S1C Fig). The number of patients with expression scores 0, 1, 2 and 3 (representing negative, weak, moderate and high expression) were 50, 35, 27 and 24 for Ang2 and $8,14,60,54$ for CD31, respectively. Ang2 expression was correlated with the vascular content of the tumours, when the Ang2 and CD31 expression scores (scores 0-3) where compared (Spearman rank order correlation test, $\mathrm{r}=0.47, P=2.3 \mathrm{e}-8$; S2A Fig). When tumours were further categorized to low and high expression categories according to Ang2 and CD31 expression scores, as explained in the methods, the CD31 high category consisted of 54 (40\%) tumours, the CD31 low category of $82(60 \%)$ tumours, Ang2 high category of 51 (38\%) tumours, and Ang2 low category of 85 (62\%) tumours (S1A and S1B Fig). High cancer Ang2 expression was strongly associated with high CD31 expression when the two distributions were compared $\left(\chi^{2}\right.$ test, $P<0.0001$ ) (S1 Table).

\section{High Baseline Endothelial Ang2 and CD31 Expression in the Tumour Are Associated with the Clinical Benefit to Sunitinib}

High pre-therapeutic Ang2 expression in the tumour vasculature was associated with a 15-percentage point increase in clinical benefit rate (CBR; partial response (PR) + stable disease (SD)) to sunitinib, when the high and low Ang2 categories were compared (CBR was 91\% in the Ang2 high category vs $76 \%$ in Ang2 low category, Fisher's exact test, $P=0.033$ ) (Fig $2 \mathrm{C}$ and $2 \mathrm{D})$. Only four (9\%) patients with high tumour Ang2 expression had progressive disease (PD) 


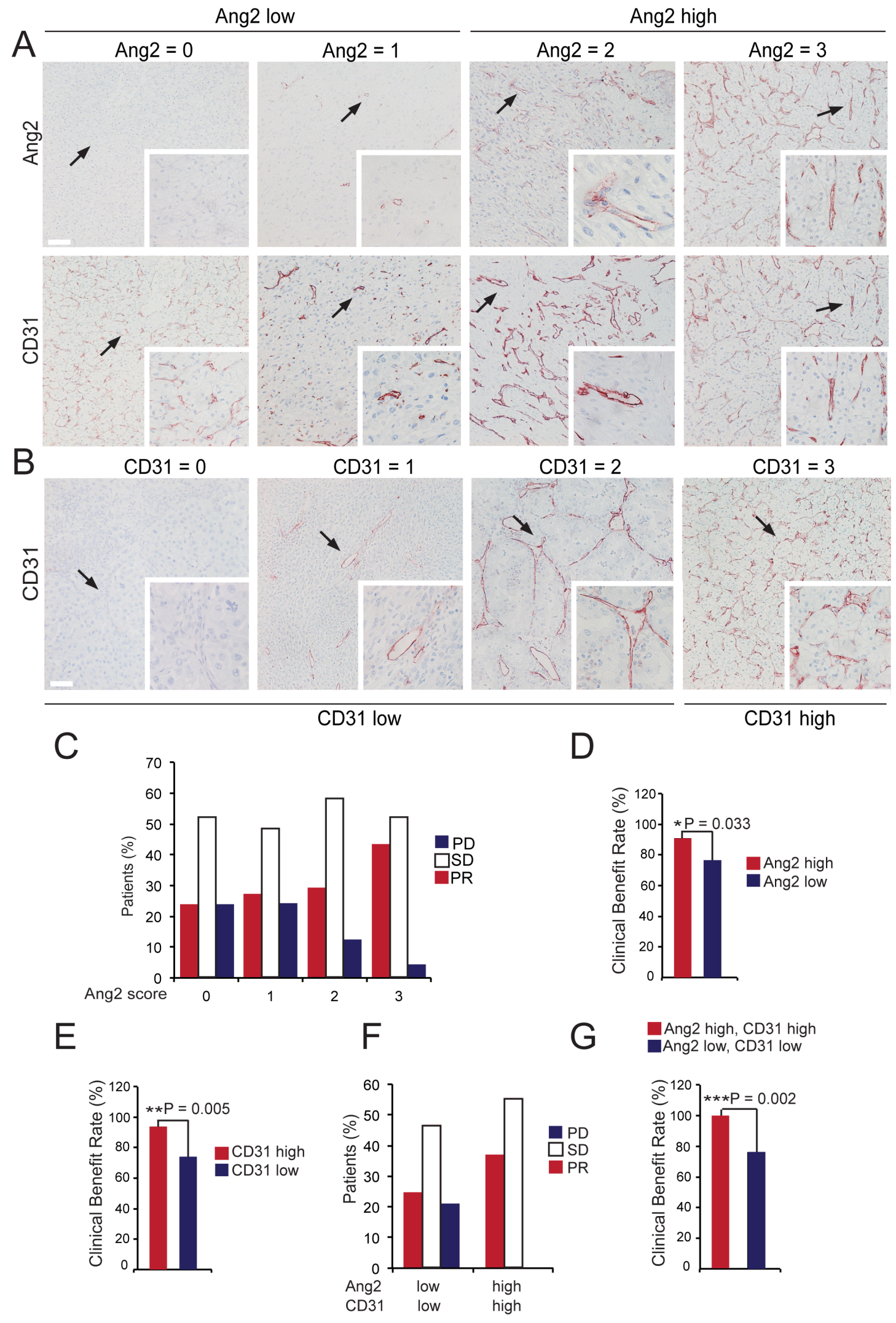


Fig 2. Association of Ang2 and CD31 expression with sunitinib response in $m R C C$. Immunohistochemical staining of $m R C C$ primary tumour tissue using antibodies to Ang2 (A) and CD31 (A, B). (A) Representative images of mRCC tumours with negative (Ang2=0), weak (Ang2 = 1), moderate $($ Ang2 = 2$)$ and high (Ang2 = 3) Ang2 expression scores, and their distribution in the Ang2 low (scores 0 and 1) and Ang2 high categories (scores 2 and 3 ) (top panel in A). Adjacent sections were stained for CD31 (bottom panel in A, scores not indicated). (B) Representative images of mRCC tumours with negative, weak, moderate $(C D 31=0, C D 31=1, C D 31=2)$ and high $(C D 31=3)$ CD31 expression scores, and their distribution in the CD31 low (scores 0-2) and CD31 high categories (score 3). (C) The percentage of patients with PR, SD and PD responses according to Ang2 expression scores $0-3$, from negative (0) to high (3) expression. (D-E) High Ang2 (D) and high CD31 (E) protein expression in the mRCC tumour vasculature was associated with increased clinical benefit rate (CBR, PR/SD sunitinib responses) using Fisher's exact test. (F) The percentage of patients with PR, SD and PD responses according to combined Ang2 and CD31 expression. (G) The combination of both high Ang2 and high CD31 protein expression was associated with increased CBR responses using Fisher's exact test. PR, partial response; SD, stabilized disease; PD, progressive disease. Arrows indicate the magnified areas. Magnifications $\times 100$ and $\times 400$. Scale bar $80 \mu \mathrm{m}$.

doi:10.1371/journal.pone.0153745.g002

as their best response to sunitinib treatment when compared to 19 (24\%) patients with low tumour Ang2 expression $(P=0.033)$. In addition, high CD31 expression was associated with a 20-percentage point increase in the CBR to sunitinib, as compared with low CD31 expression (CBR was $94 \%$ in the CD31 high category vs. $74 \%$ in the CD31 low category, Fisher's exact test, $P=0.005$ ) (Fig 2E). Only three (7\%) patients with high CD31 expression had PD as their best response as compared with $20(25 \%)$ patients with low expression of CD31 $(P=0.005)$.

Patients with both high CD31 and high Ang2 expression achieved more frequently clinical benefit with sunitinib as compared to patients with low expression of both of the markers (the CBR was 100\% in the Ang2 high and CD31 high category vs. 76\% in the Ang2 low and CD31 low category, Fisher's exact test, $P=0.002$ ) (Fig $2 \mathrm{~F}$ and $2 \mathrm{G}$ ). None of the patients with high CD31 and high Ang2 expression $(n=29)$ had progressive disease as their best response as compared with $23(24 \%)$ patients among the rest of the patients $(n=97)$.

The correlation between Ang2 and CD31 expression and clinical response was analysed based on the four Ang2 expression scores (from negative (0) to high (3) expression) and the three clinical response scores $(P R=1, S D=2, P D=3$ ), using the Spearman rank order correlation test. Pre-therapeutic Ang2, but not CD31, expression was correlated with better sunitinib response, especially with Ang2 scores 2 and 3 (Spearman rank order correlation test, $P=0.03$, S2B Fig), supporting the association of high Ang2 expression with clinical benefit to sunitinib (Fig 2D). In addition, the percentage of Ki-67 positive nuclei correlated with clinical response (Spearman rank order correlation test, $P=0.023$ ). The results were visualized in 3-dimensional scatter plots by fitting a linear surface model to the data (S2C and S2D Fig).

When the efficacy analysis was restricted to patients who received either 37.5 or $50 \mathrm{mg}$ sunitinib daily $(\mathrm{n}=88)$, high Ang2 expression and the combination of both high Ang2 and high CD31 expression were similarly associated with a high CBR (Fisher's exact test, $\mathrm{P}=0.040$ for the Ang2 high vs. Ang2 low categories, $P=0.018$ for Ang2 high and CD31 high vs. Ang2 low and $C D 31$ low category). Furthermore, high tumour Ang2 and high CD31 expression were associated with a high CBR also when the analysis was restricted to patients with clear cell RCC (Fisher's exact test, $P=0.003, \mathrm{n}=112$ ).

\section{Clinical Benefit Rate, but Not Ang2 and CD31 Expression, Is Associated with Prolonged PFS and OS}

Median PFS was 8.7 months (95\% CI 6.4 to 10.9 months) in the entire series. As expected, achieving a clinical benefit (PR/SD) correlated significantly with longer PFS (median PFS for $\mathrm{PR} / \mathrm{SD}$ patients 14.1 months and for PD patients 3.3 months, $P<0.0001$ ) and with longer OS (30.1 months for PR/SD patients vs. 12.7 months for PD patients, $P<0.0001$ ). However, Ang2 or CD31 expression had no significant association with PFS (median 11.1 months vs. 8.2 months in the Ang2 high vs. Ang2 low categories, respectively, $P=0.308$; CD31 high vs CD31 low, $P=0.851$; S2 Table), or with overall survival (22.3 vs. 22.6 months in Ang2 high vs. Ang2 
low categories, respectively, $P=0.274 ; 22.3$ vs. 22.6 months in the CD31 high and the CD31 low categories, respectively, $P=0.949 ;$ S2 Table). In line with these results, the duration of SD was not significantly longer in patients with high Ang2 and high CD31 expression (median duration of SD 8.7 months in the Ang2 high and CD31 high category) when compared to patients with low Ang2 and low CD31 expression (median SD duration 10.5 months in the Ang2 low and CD31 low category, $P=0.626$ ). Furthermore, there was no statistically significant difference in response to sunitinib, PFS or OS between patient groups receiving 50/37.5 mg vs. 25 mg sunitinib doses $(\mathrm{PFS}=9,8$ months vs. 7.8 months, $\mathrm{P}=0.167 ; \mathrm{OS}=25.2$ months vs. 22.3 months, $\mathrm{P}=0.354)$.

\section{Low Ki-67 Expression Correlates with Better Outcome}

Using $10 \%$ of Ki-67 positive nuclei (median 5) as a cut-off, high pre-therapeutic expression of Ki-67 (>10\% Ki-67 positive nuclei) was correlated with worse outcome in patients treated with sunitinib (Fig 3A-3D). In patients with more than $10 \%$ of the tumor cells expressing Ki-67 (25\% of the patients), the PFS was 6.5 months, when compared to 10.6 months in patients with low expression of Ki-67 ( $P=0.009)$ (Fig 3C). Moreover, high expression of Ki-67 predicted shortened OS in these patients (15.7 months vs. 28.5 months in the high Ki-67 vs. low Ki-67 categories, $P=0.015)$ (Fig $3 \mathrm{D})$.

\section{Discussion}

The mechanisms of both acquired and intrinsic resistance to VEGF-targeted therapy, including sunitinib, remain incompletely understood in human cancer. In addition, biomarkers predicting the clinical response to anti-angiogenic therapy are not yet available. Ang2 is known to promote tumour angiogenesis and metastasis, via signalling pathways not involving the VEGF-VEGF receptor system. Preclinical studies have identified the adaptive increase in Ang2 expression as a potential mechanism behind resistance that develops in response to anti-angiogenic therapy [12,36]. However, little information exists about Ang2 protein expression in human tumours, including RCC, about the cell types that express Ang2 in tumours and the effect of pre-therapeutic tumour Ang2 expression on the clinical response to VEGF-based antiangiogenic therapy. Our results demonstrate that high baseline Ang2, as well as CD31 expression in the primary RCC tumour vasculature was associated with an initial beneficial response to sunitinib, but not with patient survival. However, high baseline tumour cell proliferation, marked by Ki-67 expression, was strongly associated with poor outcome of patients receiving first-line sunitinib therapy, warranting further analysis of $\mathrm{Ki}-67$ as a potential biomarker of sunitinib therapy in mRCC.

The immunohistochemical method, established in the present study for the detection of Ang2, demonstrated the vascular endothelial cells, but not the RCC tumour cells, as the cell type expressing Ang2, consistently in all of the primary mRCC tumours investigated. Previous studies based on immunohistochemistry have reported Ang2 expression in the tumour cell compartment of many human cancers [37-39] and both in the endothelial and cancer cells in a smaller study based on 45 RCC samples [40]. In colorectal cancer, Ang2 mRNA was detected in the stromal, but not in the tumour cells, however, the stromal cell type expressing Ang2 was not identified [29]. Studies on mouse tumours have suggested endothelial cell-specific Ang2 mRNA expression [41], in line with the Ang2 protein analysis of 136 human mRCC tumours in this study.

Ang2 expression in the vasculature of mRCC tumour tissues, collected prior to initiation of first-line sunitinib therapy, was associated with a beneficial sunitinib response (PR/SD), whereas Ang2 expression was not correlated with OS or PFS in our study. Since patients were treated with first-line targeted therapy, subsequent treatments may have influenced the overall 
A
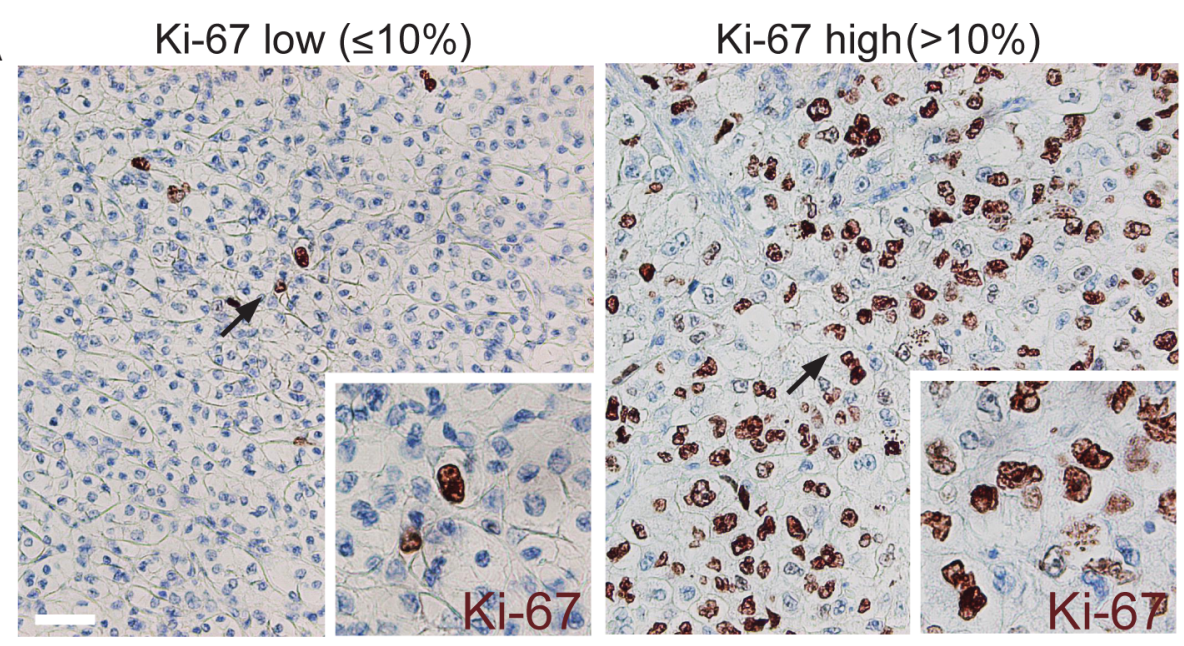

B

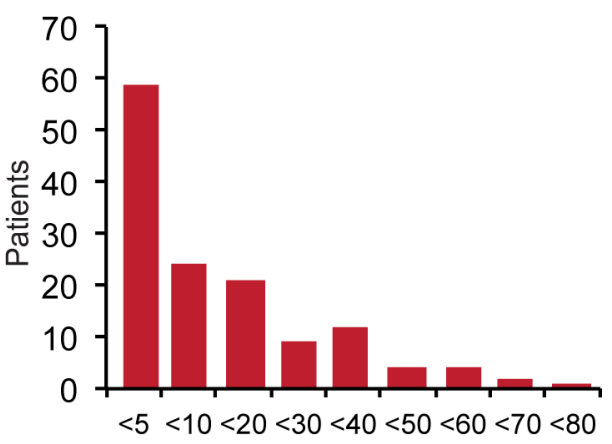

Ki-67+nuclei (\%)
C



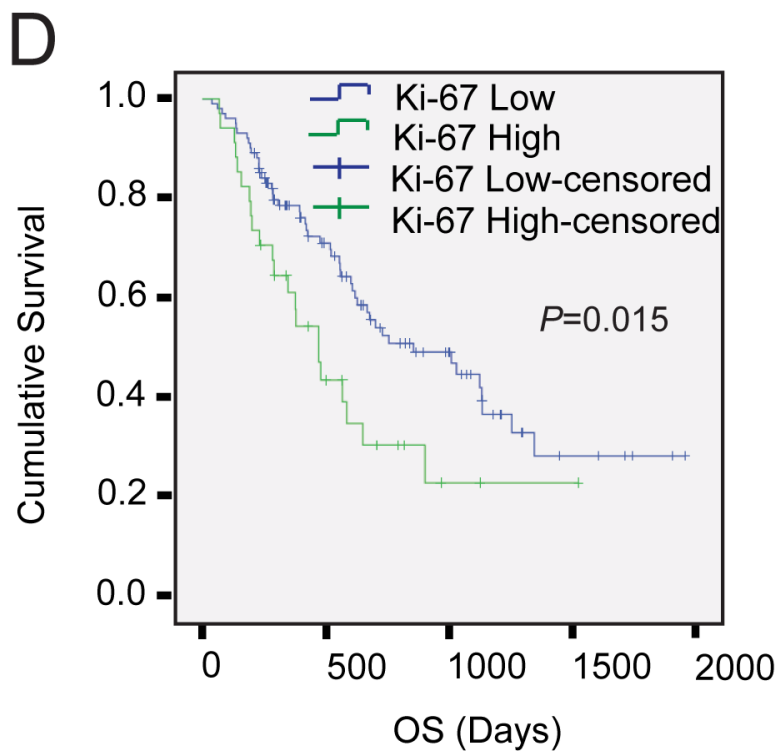

Fig 3. Correlation of Ki-67 expression with progression-free and overall survival in mRCC in response to first-line sunitinib. (A) Representative images of tumours with low Ki-67 expression (Ki-67+ nuclei $\% \leq 10 \%$ ), and high $\mathrm{Ki}-67$ expression (Ki-67+ nuclei $\%>10 \%)$. (B) The distribution of patients according to Ki-67 expression, expressed as the \% of Ki-67+nuclei. (C-D) Kaplan-Meier survival curves (Log rank) of progression-free (C) and cancer specific survival (D) in patients with low Ki-67 (blue) and high (green) Ki-67 expression scores. Arrows indicate the magnified areas. Magnifications $\times 200$ and x400. Scale bar $40 \mu \mathrm{m}$.

doi:10.1371/journal.pone.0153745.g003

survival of the patients and therefore Ang2 and CD31 expression may not have a direct influence on OS despite the association with initial treatment response. However, achieving a clinical benefit (PR/SD) was significantly associated with longer PFS and OS in the entire patient series, indicating the relevance of using CBR as a measure of sunitinib efficacy. Furthermore, Ang2 and CD31 expression were associated with clinical benefit even when the analysis was restricted to patients receiving the two highest sunitinib doses. Thus, our results support the conclusion that high baseline Ang2 expression in the blood vessels of the primary tumour does not compromise the initial sunitinib response. This result differs from the results of a recent study, which demonstrated that low baseline levels of circulating Ang2 in mRCC patients receiving first-line sunitinib predicted better outcome [30]. The observed differences may arise from the different methods used for measuring Ang2. First, it is not known whether the 
circulating Ang2 levels correlate with primary tumour-expressed Ang2 in mRCC patients. Alternatively, Ang2 expressed by metastatic nodules, or by the host vasculature as a more general response to disease, might contribute to the determined serum Ang2. Indeed, elevated circulating Ang2 levels have been reported to predict for poor prognosis in numerous noncancerous diseases, such as sepsis and malaria [16,42-44]. In these diseases, the activated endothelial cells of the normal vessels likely secrete Ang2 [45]. Second, the immunohistochemical method likely detects Ang2 stored in the Weibel-Palade bodies in endothelial cells, which may not correlate with the bioavailable Ang2 pool in the tumours. On the other hand, as Ang2 is expected to function in an autocrine manner on the vascular endothelium, the correlation of the circulating Ang2 with tumour angiogenesis remains to be investigated.

We found that endothelial Ang2 expression in the tumour vasculature correlated with the vascular density of the tumours, as determined by CD31 expression. Few studies have reported increased microvascular density, especially increased number of CD31 positive vessels, as a marker of poor prognosis in untreated ccRCC [46,47], whereas others have found that high CD31 predicts prolonged overall survival in RCC [48]. Dornbusch et al. showed that in metastatic RCC treated with sunitinib, high CD31 expression compared to low CD31 in the primary tumour was associated with better sunitinib response [49], in line with our results of the association of high CD31 with high clinical benefit rate. In addition, Puerto-Nevado et al. demonstrated using immunohistochemistry that high phospho-VEGFR2 in the tumour stroma, correlated with prolonged survival (PFS and OS) of mRCC patients treated with sunitinib [50]. These results suggest that angiogenic tumor vasculature characterized by high phosphoVEGFR-2 and Ang2 signaling is associated with beneficial sunitinib response [51].

The immunohistochemical analysis of large tumour sections in this study revealed, in the majority of the cases, intratumoural heterogeneity of the number of tumour blood vessels, Ang2 expression in the vessels and tumour cell proliferation, which is in line with intratumoural heterogeneity previously observed in mRCC [52]. Therefore, estimates for the overall vascular density, the Ang2 positive vessels counts and Ki-67 expression, were based on several areas per each tumour sample. However, we cannot exclude the effects of intratumoural heterogeneity on our results, although the large tumour sections used in this study likely provide a better estimate of overall tumour parameters than tumour microarrays.

Our results demonstrated that high baseline expression of Ki-67 predicted poor survival in mRCC patients receiving first-line sunitinib. Previously, high Ki-67 has been found to predict poor survival in local RCC, and correlate with the outcome in mRCC patients receiving immunotherapy $[33,35]$. Our results are in line with an analysis of sequential tumour samples obtained during sunitinib and pazopanib treatment of mRCC patients, which demonstrated that therapy-associated increase in Ki-67 expression correlated with poor prognosis [53].

We have analysed a relatively homogenous patient population of a moderate size consisting of consecutive patients from one academic institute. $68 \%$ of the patients received globally accepted dosing schedules and daily doses of sunitinib (37.5-50 mg/day), representing a typical non-trial patient population [34]. The main findings in our study were that Ang2 was specifically expressed in the tumour vasculature, but not in RCC tumour cells, Ang2 expression in the primary tumours correlated with tumour vascular density and that the high baseline endothelial Ang2 levels were associated with an initial beneficial response to first-line sunitinib. However, Ang2 was not associated with PFS and OS, whereas low Ki-67 was significantly associated with longer PFS and OS in patients receiving first-line sunitinib. An analysis of a different patient cohort is warranted to confirm the results obtained in this retrospective series. In conclusion, the results suggest that baseline tumour cell proliferation in the primary tumour may be considered as a potential biomarker for sunitinib efficacy, and warrants further study in series of patients treated with other anti-angiogenic agents. 


\section{Supporting Information}

S1 Fig. Distribution of patients according to Ang2 and CD31 expression scores. (A-B) The distribution of patients $(n=136)$ according to the Ang2 (A) and CD31 (B) expression scores (from negative (0) to high (3) expression), and further categorisation into the low and high expression categories. $(\mathbf{C})$ The distribution of patients according to both Ang2 and CD31 expression scores. (PDF)

S2 Fig. Correlation of Ang2, CD31, Ki-67 and response scores. (A) Spearman rank order correlation between Ang2 and CD31 expression scores, and (B) Ang2 expression and response scores. Boxplots alongside the xy-axis represent the distribution of samples based on Ang2, CD31 and response scores. Ang2 scores are distributed primarily towards the lower scores (0, 1,2 ), whereas the CD31 score distribution is towards scores 2 and 3. (A) There is a positive correlation between Ang2 and CD31 expression (Spearman rank order correlation test, $\mathrm{r}=0.47$, $P=2.3$ e-8). (B) Ang2 expression correlates with better sunitinib response (Spearman rank order correlation test, $P=0.03$ ). Green line, overall correlation; red line, locally adjusted Loess correlation; green dot, mean; green ellipses, 5\%, 25\% and 50\% data concentrations; dotted red lines, $95 \%$ confidence intervals. For clarity of the data concentrations, a small random jitter is added to visualize the data points. (C-D) 3-dimensional scatter plots with linear fitted surface (purple) of the Ang2, Ki-67 and response scores (C), and of Ang2, CD31 and response scores (D) with a smoothed linear least-squares surface fitting (green) for the data, created using the $\mathrm{R}$ package. The plots indicate the influence of decreased Ki-67 (C) and increased Ang2 expression (C-D) towards better response (lower response scores) as a decline in the purple surface towards low Ki-67+ nuclei \% (C) and Ang2 expression score 3 (C-D). Response scores: PR = 1, $\mathrm{SD}=2, \mathrm{PD}=3$; Ang2 scores 0-3; CD31 scores 0-3; from negative (0) to high (3) expression. (TIF)

\section{S1 File. Supplementary Information.}

S1 Table. Distribution of patients according to tumour Ang2 and CD31 expression scores in the Ang2 and CD31 high and low categories.

(PDF)

S2 Table. Associations between renal cell cancer Ang2 and CD31 expression and objective response rate, progression-free survival (PFS) and overall survival (OS).

(PDF)

\section{Acknowledgments}

We acknowledge Dr. Kari Alitalo for critical comments, Kirsi Mänttäri and Carita Liikanen for technical assistance, and Sami Blom and the Biomedicum Imaging Unit staff for microscopy services, respectively.

\section{Author Contributions}

Conceived and designed the experiments: PS PB. Performed the experiments: JR AL. Analyzed the data: JR AL TM AR PB PS. Contributed reagents/materials/analysis tools: AR PS PB. Wrote the paper: JR AL TM AR PB HJ PS. 


\section{References}

1. Rini BI, Campbell SC, Escudier B. Renal cell carcinoma. Lancet. 2009; 373: 1119-1132. doi: 10.1016/ S0140-6736(09)60229-4 PMID: 19269025

2. Ngo TC, Wood CG, Karam JA. Biomarkers of renal cell carcinoma. Urol Oncol. 2014; 32: 243-251. doi: 10.1016/j.urolonc.2013.07.011 PMID: 24239464

3. Chung AS, Ferrara N. Developmental and pathological angiogenesis. Annu Rev Cell Dev Biol. 2011; 27: 563-584. doi: 10.1146/annurev-cellbio-092910-154002 PMID: 21756109

4. Sato $Y$, Yoshizato T, Shiraishi Y, Maekawa S, Okuno Y, Kamura T, et al. Integrated molecular analysis of clear-cell renal cell carcinoma. Nat Genet. 2013; 45: 860-867. doi: 10.1038/ng.2699 PMID: 23797736

5. McIntyre A, Harris AL. Metabolic and hypoxic adaptation to anti-angiogenic therapy: a target for induced essentiality. EMBO Mol Med. 2015; 7: 368-379. doi: 10.15252/emmm.201404271 PMID: 25700172

6. Coppin C, Kollmannsberger C, Le L, Porzsolt F, Wilt TJ. Targeted therapy for advanced renal cell cancer (RCC): a Cochrane systematic review of published randomised trials. BJU Int. 2011; 108: 15561563. doi: 10.1111/j.1464-410X.2011.10629.x PMID: 21952069

7. Escudier B, Porta C, Schmidinger M, Algaba F, Patard JJ, Khoo V, et al. Renal cell carcinoma: ESMO Clinical Practice Guidelines for diagnosis, treatment and follow-up. Ann Oncol. 2014; 25 Suppl 3: iii4956. doi: 10.1093/annonc/mdu259 PMID: 25210086

8. Powles $T$, Chowdhury S, Jones R, Mantle M, Nathan $P, B e x A$, et al. Sunitinib and other targeted therapies for renal cell carcinoma. Br J Cancer. 2011; 104: 741-745. doi: 10.1038/sj.bjc.6606061 PMID: 21266975

9. Motzer RJ, Hutson TE, Tomczak P, Michaelson MD, Bukowski RM, Oudard S, et al. Overall survival and updated results for sunitinib compared with interferon alfa in patients with metastatic renal cell carcinoma. J Clin Oncol. 2009; 27: 3584-3590. doi: 10.1200/JCO.2008.20.1293 PMID: 19487381

10. Busch J, Seidel C, Weikert S, Wolff I, Kempkensteffen C, Weinkauf L, et al. Intrinsic resistance to tyrosine kinase inhibitors is associated with poor clinical outcome in metastatic renal cell carcinoma. BMC Cancer. 2011; 11: 295. doi: 10.1186/1471-2407-11-295 PMID: 21756335

11. Sennino B, McDonald DM. Controlling escape from angiogenesis inhibitors. Nat Rev Cancer. 2012; 12: 699-709. doi: 10.1038/nrc3366 PMID: 23001349

12. Rigamonti N, Kadioglu E, Keklikoglou I, Wyser Rmili C, Leow CC, De Palma M. Role of angiopoietin-2 in adaptive tumor resistance to VEGF signaling blockade. Cell Rep. 2014; 8: 696-706. doi: 10.1016/j. celrep.2014.06.059 PMID: 25088418

13. Sounni NE, Cimino J, Blacher S, Primac I, Truong A, Mazzucchelli G, et al. Blocking lipid synthesis overcomes tumor regrowth and metastasis after antiangiogenic therapy withdrawal. Cell Metab. 2014; 20: 280-294. doi: 10.1016/j.cmet.2014.05.022 PMID: 25017943

14. Ravaud A, Schmidinger M. Clinical biomarkers of response in advanced renal cell carcinoma. Ann Oncol. 2013; 24: 2935-2942. doi: 10.1093/annonc/mdt288 PMID: 23925998

15. Eklund L, Saharinen P. Angiopoietin signaling in the vasculature. Exp Cell Res. 2013; 319: 12711280. doi: 10.1016/j.yexcr.2013.03.011 PMID: 23500414

16. Milam KE, Parikh SM. The angiopoietin-Tie2 signaling axis in the vascular leakage of systemic inflammation. Tissue Barriers. 2015; 3: e957508. doi: 10.4161/21688362.2014.957508 PMID: 25838975

17. Fagiani E, Christofori G. Angiopoietins in angiogenesis. Cancer Lett. 2013; 328: 18-26. doi: 10.1016/j. canlet.2012.08.018 PMID: 22922303

18. Gerald D, Chintharlapalli S, Augustin HG, Benjamin LE. Angiopoietin-2: an attractive target for improved antiangiogenic tumor therapy. Cancer Res. 2013; 73: 1649-1657. doi: 10.1158/0008-5472. CAN-12-4697 PMID: 23467610

19. Wang X, Bullock AJ, Zhang L, Wei L, Yu D, Mahagaokar K, et al. The role of angiopoietins as potential therapeutic targets in renal cell carcinoma. TransI Oncol. 2014; 7: 188-195. doi: 10.1016/j.tranon. 2014.02.003 PMID: 24704536

20. Hashizume H, Falcon BL, Kuroda T, Baluk P, Coxon A, Yu D, et al. Complementary actions of inhibitors of angiopoietin-2 and VEGF on tumor angiogenesis and growth. Cancer Res. 2010; 70: 2213-2223. doi: 10.1158/0008-5472.CAN-09-1977 PMID: 20197469

21. Holopainen T, Saharinen P, D'Amico G, Lampinen A, Eklund L, Sormunen R, et al. Effects of angiopoietin-2-blocking antibody on endothelial cell-cell junctions and lung metastasis. J Natl Cancer Inst. 2012; 104: 461-475. doi: 10.1093/jnci/djs009 PMID: 22343031

22. Daly C, Eichten A, Castanaro C, Pasnikowski E, Adler A, Lalani AS, et al. Angiopoietin-2 functions as a Tie2 agonist in tumor models, where it limits the effects of VEGF inhibition. Cancer Res. 2013; 73: 108-118. doi: 10.1158/0008-5472.CAN-12-2064 PMID: 23149917 
23. Kienast $Y$, Klein C, Scheuer W, Raemsch R, Lorenzon E, Bernicke D, et al. Ang-2-VEGF-A CrossMab, a novel bispecific human IgG1 antibody blocking VEGF-A and Ang-2 functions simultaneously, mediates potent antitumor, antiangiogenic, and antimetastatic efficacy. Clin Cancer Res. 2013; 19: 67306740. doi: 10.1158/1078-0432.CCR-13-0081 PMID: 24097868

24. Mazzieri R, Pucci F, Moi D, Zonari E, Ranghetti A, Berti A, et al. Targeting the ANG2/TIE2 Axis Inhibits Tumor Growth and Metastasis by Impairing Angiogenesis and Disabling Rebounds of Proangiogenic Myeloid Cells. Cancer Cell. 2011; 19: 512-526. doi: 10.1016/j.ccr.2011.02.005 PMID: 21481792

25. Srivastava K, Hu J, Korn C, Savant S, Teichert M, Kapel SS, et al. Postsurgical adjuvant tumor therapy by combining anti-angiopoietin-2 and metronomic chemotherapy limits metastatic growth. Cancer Cell. 2014; 26: 880-895. doi: 10.1016/j.ccell.2014.11.005 PMID: 25490450

26. Monk BJ, Poveda A, Vergote I, Raspagliesi F, Fujiwara K, Bae DS, et al. Anti-angiopoietin therapy with trebananib for recurrent ovarian cancer (TRINOVA-1): a randomised, multicentre, double-blind, placebo-controlled phase 3 trial. Lancet Oncol. 2014.

27. Atkins MB, Gravis G, Drosik K, Demkow T, Tomczak P, Wong SS, et al. Trebananib (AMG 386) in Combination With Sunitinib in Patients With Metastatic Renal Cell Cancer: An Open-Label, Multicenter, Phase II Study. J Clin Oncol. 2015.

28. Sfiligoi C, de Luca A, Cascone I, Sorbello V, Fuso L, Ponzone R, et al. Angiopoietin-2 expression in breast cancer correlates with lymph node invasion and short survival. Int J Cancer. 2003; 103: 466474. PMID: 12478661

29. Goede V, Coutelle O, Neuneier J, Reinacher-Schick A, Schnell R, Koslowsky TC, et al. Identification of serum angiopoietin-2 as a biomarker for clinical outcome of colorectal cancer patients treated with bevacizumab-containing therapy. Br J Cancer. 2010; 103: 1407-1414. doi: 10.1038/sj.bjc.6605925 PMID: 20924372

30. Motzer RJ, Hutson TE, Hudes GR, Figlin RA, Martini JF, English PA, et al. Investigation of novel circulating proteins, germ line single-nucleotide polymorphisms, and molecular tumor markers as potential efficacy biomarkers of first-line sunitinib therapy for advanced renal cell carcinoma. Cancer Chemother Pharmacol. 2014; 74: 739-750. doi: 10.1007/s00280-014-2539-0 PMID: 25100134

31. Helfrich I, Edler L, Sucker A, Thomas M, Christian S, Schadendorf D, et al. Angiopoietin-2 levels are associated with disease progression in metastatic malignant melanoma. Clin Cancer Res. 2009; 15: 1384-1392. doi: 10.1158/1078-0432.CCR-08-1615 PMID: 19228739

32. Dudderidge TJ, Stoeber K, Loddo M, Atkinson G, Fanshawe T, Griffiths DF, et al. Mcm2, Geminin, and KI67 define proliferative state and are prognostic markers in renal cell carcinoma. Clin Cancer Res. 2005; 11: 2510-2517. PMID: 15814627

33. Tollefson MK, Thompson RH, Sheinin Y, Lohse CM, Cheville JC, Leibovich BC, et al. Ki-67 and coagulative tumor necrosis are independent predictors of poor outcome for patients with clear cell renal cell carcinoma and not surrogates for each other. Cancer. 2007; 110: 783-790. PMID: 17594714

34. Porta C, Paglino C, Imarisio I, Canipari C, Chen K, Neary M, et al. Safety and treatment patterns of multikinase inhibitors in patients with metastatic renal cell carcinoma at a tertiary oncology center in Italy. BMC Cancer. 2011; 11: 105. doi: 10.1186/1471-2407-11-105 PMID: 21435216

35. Gayed BA, Youssef RF, Bagrodia A, Darwish OM, Kapur P, Sagalowsky A, et al. Ki67 is an independent predictor of oncological outcomes in patients with localized clear-cell renal cell carcinoma. BJU Int. 2014; 113: 668-673. doi: 10.1111/bju.12263 PMID: 23937277

36. Keskin D, Kim J, Cooke VG, Wu CC, Sugimoto H, Gu C, et al. Targeting vascular pericytes in hypoxic tumors increases lung metastasis via angiopoietin-2. Cell Rep. 2015; 10: 1066-1081. doi: 10.1016/j. celrep.2015.01.035 PMID: 25704811

37. Nakayama T, Yoshizaki A, Kawahara N, Ohtsuru A, Wen CY, Fukuda E, et al. Expression of Tie-1 and 2 receptors, and angiopoietin-1, 2 and 4 in gastric carcinoma; immunohistochemical analyses and correlation with clinicopathological factors. Histopathology. 2004; 44: 232-239. PMID: 14987226

38. Demasi AP, Silva CA, Silva AD, Furuse $C$, Soares AB, Altemani A, et al. Expression of the vascular endothelial growth factor and angiopoietins in mucoepidermoid carcinoma of salivary gland. Head Neck Pathol. 2012; 6: 10-15. doi: 10.1007/s12105-011-0302-y PMID: 21948244

39. Li C, Fan J, Song X, Zhang B, Chen Y, Li C, et al. Expression of angiopoietin-2 and vascular endothelial growth factor receptor-3 correlates with lymphangiogenesis and angiogenesis and affects survival of oral squamous cell carcinoma. PLoS One. 2013; 8: e75388. doi: 10.1371/journal.pone.0075388 PMID: 24040410

40. Currie MJ, Gunningham SP, Turner K, Han C, Scott PA, Robinson BA, et al. Expression of the angiopoietins and their receptor Tie2 in human renal clear cell carcinomas; regulation by the von Hippel-Lindau gene and hypoxia. J Pathol. 2002; 198: 502-510. PMID: 12434420 
41. Holash J, Maisonpierre PC, Compton D, Boland P, Alexander CR, Zagzag D, et al. Vessel cooption, regression, and growth in tumors mediated by angiopoietins and VEGF. Science. 1999; 284: 19941998. PMID: 10373119

42. Parikh SM, Mammoto T, Schultz A, Yuan HT, Christiani D, Karumanchi SA, et al. Excess circulating angiopoietin-2 may contribute to pulmonary vascular leak in sepsis in humans. PLoS Med. 2006; 3: e46. PMID: 16417407

43. Kumpers $P$, van Meurs $M$, David S, Molema G, Bijzet J, Lukasz A, et al. Time course of angiopoietin-2 release during experimental human endotoxemia and sepsis. Crit Care. 2009; 13: R64. doi: 10.1186/ cc7866 PMID: 19416526

44. Conroy AL, Glover SJ, Hawkes M, Erdman LK, Seydel KB, Taylor TE, et al. Angiopoietin-2 levels are associated with retinopathy and predict mortality in Malawian children with cerebral malaria: a retrospective case-control study*. Crit Care Med. 2012; 40: 952-959. doi: 10.1097/CCM. Ob013e3182373157 PMID: 22343839

45. Fiedler U, Scharpfenecker M, Koidl S, Hegen A, Grunow V, Schmidt JM, et al. The Tie-2 ligand angiopoietin-2 is stored in and rapidly released upon stimulation from endothelial cell Weibel-Palade bodies. Blood. 2004; 103: 4150-4156. PMID: 14976056

46. Joo HJ, Oh DK, Kim YS, Lee KB, Kim SJ. Increased expression of caveolin-1 and microvessel density correlates with metastasis and poor prognosis in clear cell renal cell carcinoma. BJU Int. 2004; 93: 291-296. PMID: 14764125

47. Yao X, Qian CN, Zhang ZF, Tan MH, Kort EJ, Yang XJ, et al. Two distinct types of blood vessels in clear cell renal cell carcinoma have contrasting prognostic implications. Clin Cancer Res. 2007; 13: 161-169. PMID: 17200351

48. Virman J, Bono P, Luukkaala T, Sunela K, Kujala P, Kellokumpu-Lehtinen PL. VEGFR3 and CD31 as prognostic factors in renal cell cancer. Anticancer Res. 2015; 35: 921-927. PMID: 25667475

49. Dornbusch J, Zacharis A, Meinhardt M, Erdmann K, Wolff I, Froehner M, et al. Analyses of potential predictive markers and survival data for a response to sunitinib in patients with metastatic renal cell carcinoma. PLoS One. 2013; 8: e76386. doi: 10.1371/journal.pone.0076386 PMID: 24086736

50. del Puerto-Nevado L, Rojo F, Zazo S, Carames C, Rubio G, Vega R, et al. Active angiogenesis in metastatic renal cell carcinoma predicts clinical benefit to sunitinib-based therapy. Br J Cancer. 2014; 110: 2700-2707. doi: 10.1038/bjc.2014.225 PMID: 24786599

51. Cheng SH, Liu JM, Liu QY, Luo DY, Liao BH, Li H, et al. Prognostic role of microvessel density in patients with renal cell carcinoma: a meta-analysis. Int J Clin Exp Pathol. 2014; 7: 5855-5863. PMID: 25337227

52. Gerlinger M, Horswell S, Larkin J, Rowan AJ, Salm MP, Varela I, et al. Genomic architecture and evolution of clear cell renal cell carcinomas defined by multiregion sequencing. Nat Genet. 2014; 46: 225233. doi: $10.1038 /$ ng.2891 PMID: 24487277

53. Sharpe K, Stewart GD, Mackay A, Van Neste C, Rofe C, Berney D, et al. The effect of VEGF-targeted therapy on biomarker expression in sequential tissue from patients with metastatic clear cell renal cancer. Clin Cancer Res. 2013; 19: 6924-6934. doi: 10.1158/1078-0432.CCR-13-1631 PMID: 24130073 